\title{
A teenager presenting with a forearm mass
}

\author{
Rachel Kopkin $^{1} \cdot$ Carlos Gimenez $^{2}$ - Ernest Rudman ${ }^{2}$
}

Published online: 1 August 2017

(C) ISS 2017

\section{Reversed palmaris longus}

The palmaris longus is a muscle of the upper extremity that exhibits a wide spectrum of anatomical variants, more so than other muscles of the upper extremity. In its most common form it is a small, fusiform muscle that functions as a vestigial flexor of the wrist and originates at the medial epicondyle as part of the common flexor tendon and inserts at the palmar aponeurosis volar to the flexor retinaculum [1]. It receives its vascular supply from branches of the ulnar and brachial arteries and is innervated by the median nerve [1].

Several anatomic variants of the palmaris longus are recognized with the most common being agenesis affecting 2$25 \%$ of the population [1]. A reversed palmaris longus and the remaining variants (bifid, triple headed, accessory palmaris longus and palmaris profundus) are found in approximately $9 \%$ of the population [1]. Awareness of such muscular anomalies is important because they may cause symptoms of

The case presentation can be found at doi: 10.1007/s00256-017-2717-3

Ernest Rudman

erudman@gmail.com

1 Louisiana State University Health Sciences Center School of Medicine, 1901 Perdido Street, New Orleans, LA 70112, USA

2 MRI/MSK Division, Department of Radiology, Ochsner Clinic Foundation, 1514 Jefferson Highway, New Orleans, LA 70121, USA

median and possibly ulnar nerve entrapment, which can be indistinguishable [2].

Ultrasound, CT and MRI are useful in the evaluation of anomalous muscles and can help patients avoid unnecessary surgery. A reversed palmaris longus demonstrates a tendinous origin at the medial epicondyle that becomes more muscular two thirds down the forearm becoming tendinomembranous at the palmar aponeurosis. T1-weighted MR images reveal a pseudomass that is isointense to muscle just medial to the flexor carpi radialis tendon (Fig. 1, 3) in the expected location of the palmaris longus tendon [2,3]. This pseudomass remains isointense to muscle on STIR images (Fig. 2).

In patients with effort-related compartment syndrome of the forearm due to a reversed or hypertrophied palmaris longus, activity-related increased blood flow leads to increased intramuscular pressure. Because the antebrachial fascia prevents expansion in the direction of the skin, the hyperemic anomalous muscle will excerpt pressure inwardly causing compression of the surround structures, including the median nerve proximal to the carpal tunnel. Patient's typically report pain and tingling in the fingers following sports or manual exercises [4]. Muscle atrophy is uncommon but can occur with chronic or severe compression $[4,5]$. Symptomatic patients can be treated with surgical excision of the anomalous muscle without significant functional loss. The above patient underwent no intervention. As of March 2017, the pseudomass remained without significant symptomatology. 


\section{Compliance with ethical standards}

Conflict of interest The authors declare that they have no conflict of interest.

\section{References}

1. Murabit A, Gnarra M, Mohamed A. Reversed Palmaris longus: anatomical variant - case report and literature review. Can J Plast Surg. 2013;21(1):55-6.
2. Polesuk BS, Helms CA. Hypertrophied palmaris longus muscle, a pseudomass of the forearm: MR appearance - case report and review of the literature. Radiology. 1998;207:361-2.

3. Timins M. Muscular anatomic variants of the wrist and hand: findings on MR imaging. AJR. 1999;172:1397-401.

4. Schuurman H, van Gils APG. Reversed Palmaris longus muscle on MRI: report of four cases. Eur Radiol. 2000;10(8):1242-4.

5. Linda D, Harish S, Stewart B, Finlay K, Parasu N, Rebello R. Multimodality imaging of peripheral neuropathies of the upper limb and brachial plexus. Radiographics. 2010;30:1373-400. 